\title{
A computer program to generate parametric and nonparametric signal-detection parameters
}

\author{
RUSSEL BOICE and RICK M. GARDNER \\ University of Southern Colorado, Pueblo, Colorado
}

(Paul Schnur, Sponsor)

\begin{abstract}
A computer program is described that calculates parametric and nonparametric signal-detection parameters for sensory sensitivity and response bias. Advantages of this program over previously published programs are discussed.
\end{abstract}

Signal-detection theory (SDT) is a psychophysical methodology originally proposed by Tanner and Swets (1954) that serves as an alternative to classical psychophysical methodologies. One advantage that it offers is the separate determination of parameters reflecting sensory and nonsensory factors. These factors are confounded with classical psychophysical methods.

In SDT, the sensitivity of the decision-making process is a function of the variable $d^{\prime}$, which is equal to the distance between the noise and the signal-plus-noise distribution and which is monotonically related to the signal strength. Nonsensory factors are reflected in the response criterion. The most commonly used measure of response criterion is $\beta$, which is defined as the ratio of the ordinate of the signal-plus-noise distribution at the criterion to the ordinate of the noise distribution.

Several computer programs have been developed in recent years to generate SDT parameters. In an earlier article (Gardner \& Boice, 1986), we described a computer program that accurately computes measures of sensory sensitivity and response bias. We now describe a revised version of this program that has greater accuracy and that additionally generates nonparametric SDT indexes for sensitivity and bias.

The development of signal-detection theory has rested on specific assumptions about the underlying distribution of noise and signal plus noise. When information about the distributions is not known, data are often judged by how close they lie to operating characteristics derived from normal distributions. In recent years, there has been a growing interest in various nonparametric analyses of detection experiments in which specific underlying distributions are not assumed. Nonparametric estimates also

This research was supported by Grant 5 S06 RR08197 to R. M. Gardner funded by the Division of Research Resources and the National Institute of Mental Health. Turbo Pascal is a trademark of and is available from Borland International, 4585 Scotts Valley Dr., Scotts Valley, CA 95066. Requests for reprints should be sent to Rick M. Gardner, Department of Psychology, University of Southern Colorado, Pueblo, CO 81001 . are useful under conditions in which a relatively small number of trials are presented, as is often the case in clinical studies (Grossberg \& Grant, 1978).

Pollack and Norman (1964) and Hodos (1970) proposed nonparametric SDT measures based on the geometry of the unit square that can be interpreted as indexes of sensitivity and bias. Neither paper gave functional expressions for computing the indexes from the data. Grier (1971) later provided such computing expressions for the indexes and for their associated isosensitivity and isobias contours, and showed the relationship of the nonparametric sensitivity index to the area measure.

We describe a program (see Appendix) that computes both parametric and nonparametric sensitivity and bias measures. The program is written in Turbo Pascal and runs on any computer that runs Turbo Pascal, including Apple, IBM, Tandy, Sperry, and any other CPM or MSDOS compatible computer.

The inverse normal distribution area function is needed to calculate parametric measures of sensory sensitivity and response bias. This function (implemented as function $f$ in the program) is approximated using an algorithm outlined by Odeh and Evans (1974). Brophy (1985) reviewed this and other algorithms and reported an accuracy of six significant digits for the Odeh and Evans algorithm. The normal distribution function is used in calculating $\beta$ and is implemented as function $g$ in the program.

After entering the proportion of hits and false alarms, the program generates the following SDT parameters: (1) $d^{\prime}-$ A parametric measure of sensory sensitivity equal to the distance between the noise and the signal-plus-noise distribution. (2) $\beta-A$ parametric measure of response bias, defined as the ratio of the ordinate of the signal-plusnoise distribution at the criterion to the ordinate of the noise distribution. (3) $\ln \beta-\mathrm{A}$ parametric measure of response bias that can be averaged over subjects, trial blocks, and so forth. The rationale for this log transformation of $\beta$ was given by Gardner and Boice (1986). (4) $A^{\prime}-$ A commonly used nonparametric index of sensory sensitivity developed by Pollack and Norman (1964). (5) $\beta^{\prime}-\mathrm{A}$ nonparametric measure of bias suggested by 
Hodos (1970). Hodos (1970) gave a detailed explanation of the above-mentioned nonparametric indexes for sensitivity and bias.

One feature of the present program is that it calculates values of sensory sensitivity and response bias, even if individual subjects' hit or false-alarm rates contain proportions of 0 or 1 , which correspond to infinite $Z$ scores for computing values of $d^{\prime}$. In these circumstances, the program follows the procedure outlined by Macmillan and Kaplan (1985), in which 0 is replaced by $1 /(2 N)$ and 1 is replaced by $1-1 /(2 N)$, where $N$ is the number of trials on which the relevant stimulus was presented. When a 0 or a 1 is entered as a data point, the program requests $N$.

This program has two advantages over previously published programs: accuracy is increased to five significant digits, and the results are displayed instantly. The simultaneous generation of parametric and nonparametric sensitivity and bias measures should prove helpful to experimenters who wish to compare SDT values with differing underlying assumptions regarding the noise and signal-plus-noise distributions.

\section{REFERENCES}

Brophy, A. L. (1985). Approximation of the inverse normal distribution function. Behavior Research Methods, Instruments, \& Computers, 17, 415-417.

GaRdNer, R. M., \& Boice, R. (1986). A computer program to generate signal-detection theory values for sensitivity and response bias. Behavior Research Methods, Instruments, \& Computers, 18, 54-56.

Grier, J. B. (1971). Nonparametric indexes for sensitivity and bias: Computing formulas. Psychological Bulletin, 75, 424-429.

Grossberg, J. M., \& Grant, B. F. (1978). Clinical psychophysics: Applications of ratio scaling and signal detection methods to research on pain, fear, drugs, and medical decision making. Psychological Bulletin, 85, 1154-1176.

HoDos, W. (1970). A nonparametric index of response bias for use in detection and recognition experiments. Psychological Bulletin, 74, 351-354.

Macmillan, N. A., \& Kaplan, H. L. (1985). Detection theory analysis of group data: Estimating sensitivity from average hit and falsealarm rates. Psychological Bulletin, 98, 185-199.

Odeh, R. E., \& Evans, J. O. (1974). Algorithm AS 70: The percentage points of the normal distribution. Applied Statistics, 23, 96-97.

Pollack, I., \& Norman, D. A. (1964). A nonparametric analysis of recognition experiments. Psychonomic Science, 1, 125-126.

TANNER, W. P., \& SWETs, J. A. (1954). A decision-making theory of visual detection. Psychological Review, 61, 401-409.

\section{APPENDIX}

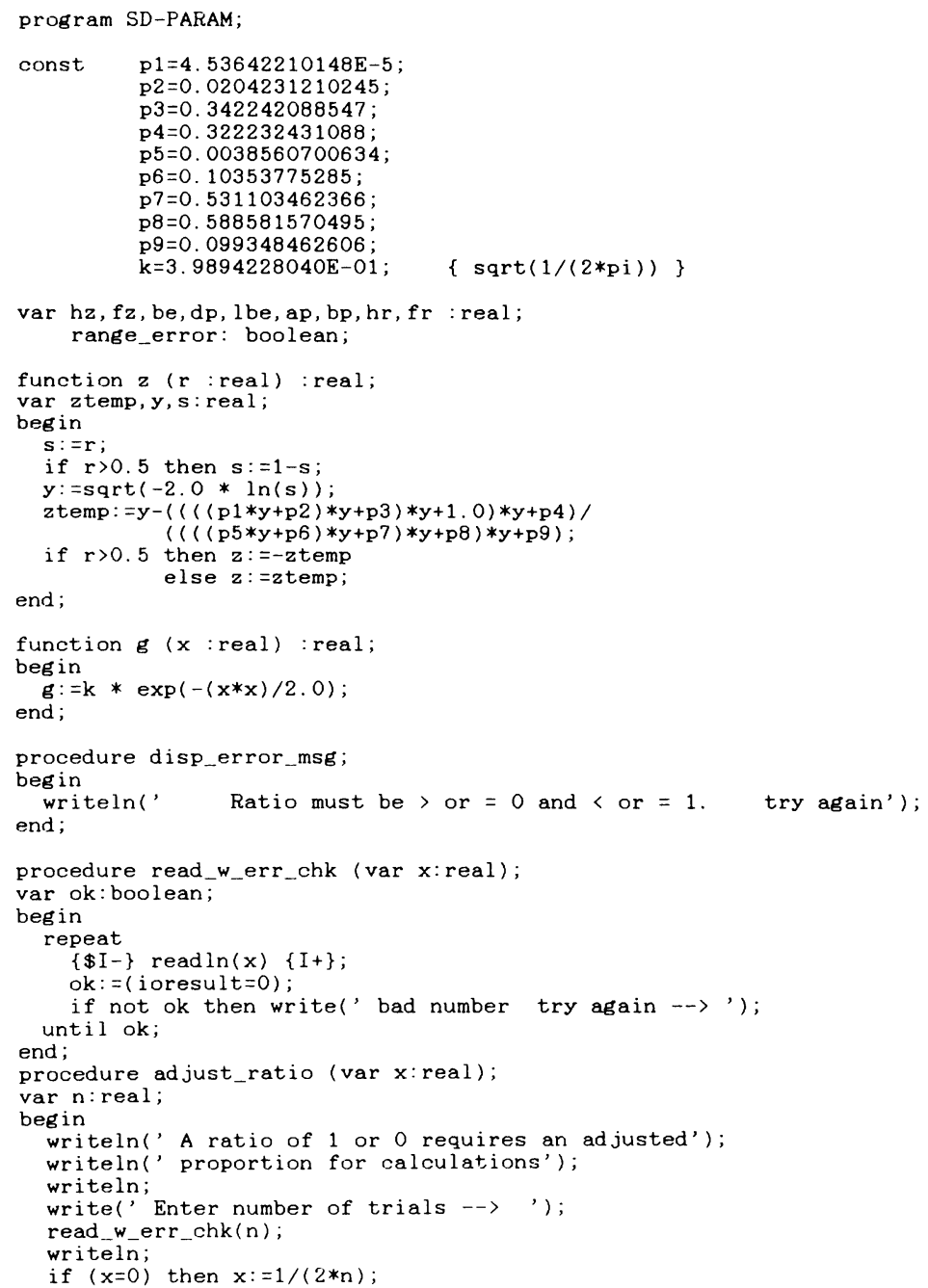


APPENDIX (Continued)

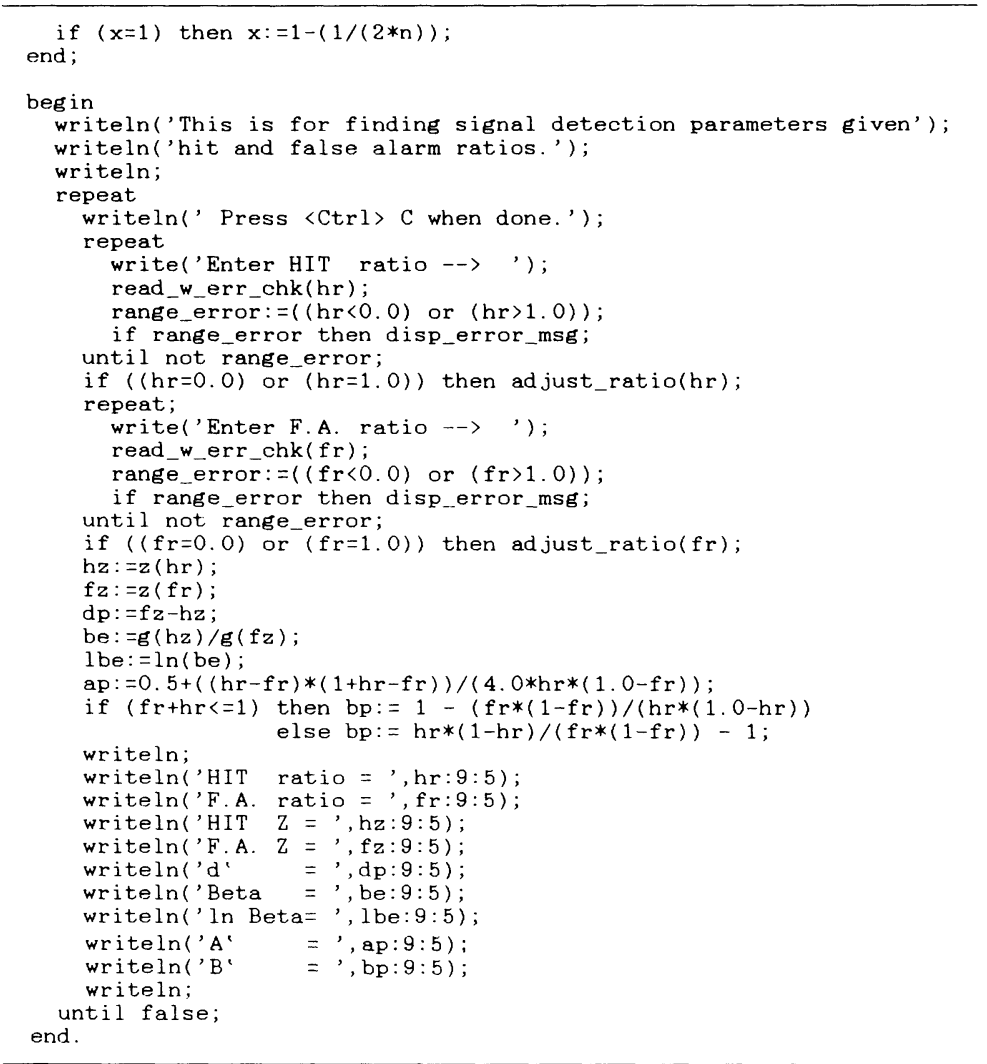

(Manuscript received for publication January 11, 1988.) 